\title{
Berichtigungen.
}

\author{
$\mathrm{Zu} \mathrm{Band} 40$ :
}

S. 649 , Zeile 9 v. o. soll lauten:, ,ist $\alpha a^{-1}$ anf Grund der Wahl der $\alpha_{i}$ zu $b_{1}$ teilerfremd, also".

S. 702, Zeile 4 v. o. fehlt auf der rechten Seite der Gleichung der Faktor: $(1-x)^{-2 \beta}$.

S. 702 , Zeile 7 u. 8 v. o.: statt $(\lambda+2 \alpha i)^{-2 p-1}$, soll es heißen $\lambda^{-2 p-1}$, statt $(\lambda-\alpha i)^{2}$ soll es heißen $\lambda^{2}$.

\section{Zu Band 41 :}

S. 486 ist die folgende Fußnote anzufügen:

„Eine Buschenveloppe $B_{r}(r>3)$ hat mindestens einen Doppelpunkt, weil eine ebene Kurve mit Rückkehrpunkten erster Art ohne andere Punktoder Tangentensingularitäten genau drei Rückkehrpunkte besitzt. S. Fußnote 12$). "$

S. 681 soll die rechte Seite Gleichung (4) lauten: $\frac{\pi}{2} J_{n+\frac{1}{2}}^{2}(z)$.

S. 685 , Zeile 2 v. u.: statt „unrestrided" lies "unrestricted".

\section{Zu Band 42:}

S. 54, Zeile 5 v. o.: statt $p+p^{\prime \prime}$ lies $p+k p^{\prime \prime}$.

S. 56 , Zeile 7 v. o.: statt $\Pi$ lies $\Sigma$.

S. 134 in Gleichung $(2,12)$ soll es $e^{-\frac{1}{2 s}}$ statt $e^{\frac{1}{2 s}}$ heißen.

S. 255 , Zeile 4 v. u.: statt $a$, $a, a^{k^{2}}, \ldots$ soll es heißen $a, a^{k}, a^{k^{2}}, \ldots$.

S. 352, Zeile 5 v. o. : statt $x_{1}=t$ soll es heißen $X_{1}=t$.

S. 440 , Zeile 8 v. u.: statt Vollständigkeit soll es heißen Vollstetigkeit.

S. 441, Zeile 2 v. u. : statt S. 39 soll es heißen S. 468.

S. 448, Zeile 3 v. o.: statt $|x|>x^{*}$ soll es heißen $|x|<x^{*}$.

S. 478 , Zeile 16 v. u.: statt $\varphi\left(x, y_{1}\right)$ soll es heißen $\varphi\left(x, \lambda_{1}\right)$. 